PROCEEDINGS OF THE

AMERICAN MATHEMATICAL SOCIETY

Volume 138, Number 11, November 2010, Pages 3849-3862

S 0002-9939(2010)10417-3

Article electronically published on May 24, 2010

\title{
A CHARACTERIZATION OF CLOSURE OPERATIONS THAT INDUCE BIG COHEN-MACAULAY MODULES
}

\author{
GEOFFREY D. DIETZ
}

(Communicated by Bernd Ulrich)

\begin{abstract}
The intent of this paper is to present a set of axioms that are sufficient for a closure operation to generate a balanced big Cohen-Macaulay module $B$ over a complete local domain $R$. Conversely, we show that if such a $B$ exists over $R$, then there exists a closure operation that satisfies the given axioms.
\end{abstract}

In equal characteristic, the tight closure operation has been used to present proofs of the existence of balanced big Cohen-Macaulay modules and algebras. (See [D, Ho1, [Ho2, and [HH3] for example.) Finding a closure operation in mixed characteristic that is powerful enough to produce big Cohen-Macaulay modules or algebras has been an elusive yet very important goal for some time now, as the existence of such a closure operation would yield major results related to the homological conjectures in commutative algebra.

In this article, we present a list of seven axioms for a closure operation defined for finitely generated modules over a complete local domain $R$. After deriving some simpler consequences of the axioms (including colon-capturing), we prove that a closure operation satisfying the axioms implies the existence of a balanced big Cohen-Macaulay module over $R$. A balanced big Cohen-Macaulay module $B$ over $(R, \mathfrak{m})$ is an $R$-module where every system of parameters in $R$ is a regular sequence on $B$ (i.e., if $x_{1}, \ldots, x_{k+1}$ forms part of a system of parameters in $R$ and $b \in\left(x_{1}, \ldots, x_{k}\right):_{B} x_{k+1}$, then $\left.b \in\left(x_{1}, \ldots, x_{k}\right) B\right)$ and $\mathfrak{m} B \neq B$. Our main tools for proving this theorem will be Hochster's method of modifications Ho1 and the use of analogues of phantom extensions developed by Hochster and Huneke [HH3].

We then show that the existence of a balanced big Cohen-Macaulay module over a complete local domain can be used to create a closure operation that satisfies all of the axioms.

Finally, we demonstrate that all of the axioms are satisfied by tight closure. We also examine the axioms in relation to several other common closure operations associated with tight closure theory.

\section{The Closure AXIOMS AND CONSEQUences}

Let $R$ be a complete local domain, and let $N \subseteq M$ be finitely generated modules. An operation satisfying Axioms (1)-(5) below will be called a closure operation and will be denoted by $N_{M}^{\natural}$ for the closure of $N$ within $M$. Throughout the paper we will

Received by the editors October 28, 2009 and, in revised form, January 30, 2010.

2000 Mathematics Subject Classification. Primary 13C14; Secondary 13A35.

(C)2010 American Mathematical Society 3849

Reverts to public domain 28 years from publication 
call this the $t$-closure of $N$ in $M$. In Section 5 , we will show that tight closure, plus closure, Frobenius closure, and solid closure all satisfy Axioms (1)-(6), while tight, plus, and solid closures (at least in positive characteristic) also satisfy Axiom (7).

Axioms 1.1. Let $(R, \mathfrak{m})$ be a fixed complete local domain. Let $I$ be an arbitrary ideal of $R$, and let $N, M$, and $W$ be arbitrary finitely generated $R$-modules with $N \subseteq M$.

(1) $N_{M}^{\natural}$ is a submodule of $M$ containing $N$.

(2) $\left(N_{M}^{\natural}\right)_{M}^{\natural}=N_{M}^{\natural}$; i.e., the $\natural$-closure of $N$ in $M$ is closed in $M$.

(3) If $N \subseteq M \subseteq W$, then $N_{W}^{\natural} \subseteq M_{W}^{\natural}$.

(4) Let $f: M \rightarrow W$ be a homomorphism. Then $f\left(N_{M}^{\natural}\right) \subseteq f(N)_{W}^{\natural}$.

(5) If $N_{M}^{\natural}=N$, then $0_{M / N}^{\natural}=0$.

(6) The ideals $\mathfrak{m}$ and 0 are $\natural$-closed; i.e., $\mathfrak{m}_{R}^{\natural}=\mathfrak{m}$ and $0_{R}^{\natural}=0$.

(7) Let $x_{1}, \ldots, x_{k+1}$ be a partial system of parameters for $R$, and let $J=$ $\left(x_{1}, \ldots, x_{k}\right)$. Suppose that there exists a surjective homomorphism $f: M \rightarrow$ $R / J$ and $v \in M$ such that $f(v)=x_{k+1}+J$. Then

$$
(R v)_{M}^{\natural} \cap \operatorname{ker} f \subseteq(J v)_{M}^{\natural} .
$$

The final axiom will be referred to as the generalized colon-capturing property due to the fact that we will derive ordinary colon-capturing from it.

The following is a list of basic properties of a closure operation that mimics part of the list given for tight closure of modules in [HH1, Section 8].

Lemma 1.2. Let $R$ be a complete local domain possessing a closure operation that satisfies Axioms (1)-(5). In the following, $N, N^{\prime}$, and $N_{i} \subseteq M_{i}$ are all $R$ submodules of the finitely generated $R$-module $M$.

(a) Let $N^{\prime} \subseteq N \subseteq M$. Then $u \in N_{M}^{\natural}$ if and only if $u+N^{\prime} \in\left(N / N^{\prime}\right)_{M / N^{\prime}}^{\natural}$.

(b) If $\mathcal{I}$ is a finite set, $N=\bigoplus_{i \in \mathcal{I}} N_{i}$, and $M=\bigoplus_{i \in \mathcal{I}} M_{i}$, then we have $N_{M}^{\natural}=$ $\bigoplus_{i \in \mathcal{I}}\left(N_{i}\right)_{M_{i}}^{\natural}$

(c) Let $\mathcal{I}$ be any set. If $N_{i} \subseteq M$ for all $i \in \mathcal{I}$, then $\left(\bigcap_{i \in \mathcal{I}} N_{i}\right)_{M}^{\natural} \subseteq \bigcap_{i \in \mathcal{I}}\left(N_{i}\right)_{M}^{\natural}$.

(d) Let $\mathcal{I}$ be any set. If $N_{i}$ is $\natural$-closed in $M$ for all $i \in \mathcal{I}$, then $\bigcap_{i \in \mathcal{I}} N_{i}$ is 4 -closed in $M$.

(e) $\left(N_{1}+N_{2}\right)_{M}^{\natural}=\left(\left(N_{1}\right)_{M}^{\natural}+\left(N_{2}\right)_{M}^{\natural}\right)_{M}^{\natural}$.

Proof.

(a) Since $\left(M / N^{\prime}\right) /\left(N / N^{\prime}\right) \cong M / N$, it is enough to prove the assertion for the case where $N^{\prime}=0$. Let $\pi: M \rightarrow M / N$ be the natural surjection. If $u \in N_{M}^{\natural}$, then Axiom (4) implies that $u+N=\pi(u) \in(\pi(N))_{M / N}^{\natural}=0_{M / N}^{\natural}$. Conversely, suppose that $u+N \in 0_{M / N}^{\natural}$. By Axiom (1), there is a natural homomorphism $M / N \rightarrow M / N_{M}^{\natural}$. Applying Axiom (4) to this map yields $u+N_{M}^{\natural} \in 0_{M / N_{M}^{\natural}}^{\natural}$. By Axiom (2) and Axiom (5), 0 is $\natural$-closed in $M / N_{M}^{\natural}$, so $u+N_{M}^{\natural}$ is zero in the quotient $M / N_{M}^{\natural}$.

(b) Let $\pi_{i}: M \rightarrow M_{i}$ be the natural projection, and let $\iota_{i}: M_{i} \rightarrow M$ be the natural inclusion for all $i$. By Axiom (4),

$$
\pi_{i}\left(N_{M}^{\natural}\right) \subseteq \pi_{i}(N)_{M_{i}}^{\natural}=\left(N_{i}\right)_{M_{i}}^{\natural} .
$$


Therefore, $N_{M}^{\natural} \subseteq \bigoplus_{i}\left(N_{i}\right)_{M_{i}}^{\natural}$ in $M=\bigoplus_{i} M_{i}$. Conversely, we apply Axiom (4) to the map $\iota_{i}$ to see that $\iota_{i}\left(\left(N_{i}\right)_{M_{i}}^{\natural} \subseteq \iota_{i}\left(N_{i}\right)_{M}^{\natural}\right.$, which is contained in $N_{M}^{\natural}$ by Axiom (3). Thus, $\bigoplus_{i}\left(N_{i}\right)_{M_{i}}^{\natural} \subseteq N_{M}^{\natural}$.

(c) Let $u \in\left(\bigcap_{i \in \mathcal{I}} N_{i}\right)_{M}^{\natural}$. By Axiom (3), $u \in\left(N_{i}\right)_{M}^{\natural}$ for all $i \in \mathcal{I}$.

(d) Since each $N_{i}$ is -closed, the conclusion follows from (c) and Axiom (1).

(e) As $N_{i} \subseteq\left(N_{i}\right)_{M}^{\natural}$ by Axiom (1), Axiom (3) implies that

$$
\left(N_{1}+N_{2}\right)_{M}^{\natural} \subseteq\left(\left(N_{1}\right)_{M}^{\natural}+\left(N_{2}\right)_{M}^{\natural}\right)_{M}^{\natural} .
$$

Conversely, $N_{i} \subseteq N_{1}+N_{2}$, with Axiom (3), implies that $\left(N_{i}\right)_{M}^{\natural} \subseteq\left(N_{1}+\right.$ $\left.N_{2}\right)_{M}^{\natural}$. Therefore, $\left(N_{1}\right)_{M}^{\natural}+\left(N_{2}\right)_{M}^{\natural} \subseteq\left(N_{1}+N_{2}\right)_{M}^{\natural}$. Axiom (2) then yields

$$
\left(\left(N_{1}\right)_{M}^{\natural}+\left(N_{2}\right)_{M}^{\natural}\right)_{M}^{\natural} \subseteq\left(\left(N_{1}+N_{2}\right)_{M}^{\natural}\right)_{M}^{\natural}=\left(N_{1}+N_{2}\right)_{M}^{\natural} \text {. }
$$

Although we only need Axiom (7) in the form given in order to produce a balanced big Cohen-Macaulay module, we can derive a more general property that does not require the map $M \rightarrow R / J$ to be surjective. In fact, if an operation satisfies Axioms (1), (3), and (4), then Axiom (7) is equivalent to Lemma 1.3 .

Lemma 1.3. Let $R$ be a complete local domain possessing an operation that satisfies Axioms (1), (3), (4), and (7). Let $x_{1}, \ldots, x_{k+1}$ be a partial system of parameters for $R$, and let $J=\left(x_{1}, \ldots, x_{k}\right)$. Suppose that there exists a homomorphism $f$ : $M \rightarrow R / J$ such that $f(v)=x_{k+1}+J$. Then

$$
(R v)_{M}^{\natural} \cap \operatorname{ker} f \subseteq(J v)_{M}^{\natural} .
$$

Proof. Given the setup above, suppose that $u \in(R v)_{M}^{\natural} \cap \operatorname{ker} f$. Define a new homomorphism $\phi: M \oplus R \rightarrow R / J$ by $\phi(m, r)=f(m)+r+J$. The map $\phi$ is surjective as $\phi(0,1)=1+J$. Also, $\phi(v, 0)=f(v)=x_{k+1}+J$. Therefore, $\phi$ satisfies the assumptions of Axiom (7), and we may conclude that

$$
(R(v, 0))_{M \oplus R}^{\natural} \cap \operatorname{ker} \phi \subseteq(J(v, 0))_{M \oplus R}^{\natural} .
$$

Lemma 1.2(b) implies that $(R(v, 0))_{M \oplus R}^{\natural}=(R v)_{M}^{\natural} \oplus 0_{R}^{\natural}$ and that $(J(v, 0))_{M \oplus R}^{\natural}=$ $(J v)_{M}^{\natural} \oplus 0_{R}^{\natural}$. Thus, $(u, 0) \in(J v)_{M}^{\natural} \oplus 0_{R}^{\natural}$, and so $u \in(J v)_{M}^{\natural}$ as desired.

We can now derive a colon-capturing property analogous to one found in tight closure theory.

Proposition 1.4 (colon-capturing). Let $R$ be as in the previous lemma. Let $x_{1}, \ldots, x_{k+1}$ be a partial system of parameters in $R$. Then $\left(x_{1}, \ldots, x_{k}\right): x_{k+1} \subseteq$ $\left(x_{1}, \ldots, x_{k}\right)_{R}^{\natural}$.

Proof. Apply the hypotheses above to Lemma 1.3 with $M=R, J=\left(x_{1}, \ldots, x_{k}\right)$, $f: R \rightarrow R / J$ given by $f(r)=r x_{k+1}+J$, and $v=1$. Then $f(1)=x_{k+1}+J$, $(R v)_{M}^{\natural}=R$ by Axiom (1), ker $f=J: x_{k+1}$, and $(J v)_{M}^{\natural}=J_{R}^{\natural}$.

\section{2. দ-PHANTOM EXTENSIONS}

In this section we define a notion of phantom extensions for a closure operation satisfying Axioms (1)-(5). Phantom extensions and module modifications were used in $\underline{\mathrm{HH} 3}$ to produce a new proof of the existence of big Cohen-Macaulay modules in positive characteristic. Our study of $\lfloor$-phantom extensions will lead to the existence of a balanced big Cohen-Macaulay module over $R$. 
In [HH3, Section 5], a map $\alpha: N \rightarrow M$ of finitely generated $R$-modules is a phantom extension if there exists $c \in R$ but not in any minimal prime such that for all $e \gg 0$, there exists $\gamma_{e}: \mathbf{F}^{e}(M) \rightarrow \mathbf{F}^{e}(N)$ such that $\gamma_{e} \circ \mathbf{F}^{e}(\alpha)=c\left(\operatorname{id}_{\mathbf{F}^{e}(N)}\right)$, where $\mathbf{F}^{e}$ is the iterated Frobenius functor.

Via the Yoneda correspondence, every short exact sequence

$$
0 \rightarrow N \stackrel{\alpha}{\rightarrow} M \rightarrow Q \rightarrow 0
$$

corresponds to a unique element $\epsilon$ of $\operatorname{Ext}_{R}^{1}(Q, N)$. Let $P_{\bullet}$ be a projective resolution of $Q=M / \alpha(N)$. Then $\operatorname{Ext}_{R}^{1}(Q, N)$ is isomorphic to $H^{1}\left(\operatorname{Hom}_{R}\left(P_{\bullet}, N\right)\right)$.

Given the map $\alpha: N \rightarrow M$ and the corresponding element $\epsilon$ of $\operatorname{Ext}_{R}^{1}(Q, N)$, Hochster and Huneke called $\epsilon$ a phantom element if a cocycle representative of $\epsilon$ in $\operatorname{Hom}_{R}\left(P_{1}, N\right)$ is in the tight closure of

$$
\operatorname{Im}\left(\operatorname{Hom}_{R}\left(P_{0}, N\right) \rightarrow \operatorname{Hom}_{R}\left(P_{1}, N\right)\right)
$$

within $\operatorname{Hom}_{R}\left(P_{1}, N\right)$. In the case where $N=R$, Hochster and Huneke provide the following equivalence.

Theorem 2.1 (Theorem 5.13, $\mathrm{HH} 3$ ). Let $R$ be a reduced Noetherian ring of positive characteristic. An exact sequence

$$
0 \rightarrow R \stackrel{\alpha}{\rightarrow} M \rightarrow Q \rightarrow 0
$$

is a phantom extension if and only if the corresponding element $\epsilon$ in $\operatorname{Ext}_{R}^{1}(Q, R)$ is phantom in the sense described above.

We will use this latter property to define $\downarrow$-phantom extensions with respect to Ł-closure.

Definition 2.2. Let $R$ be a complete local domain possessing a closure operation satisfying Axioms (1)-(5). Let $M$ be a finitely generated $R$-module and $\alpha: R \rightarrow M$ an injective $R$-linear map. With $Q=M / \alpha(R)$, we have an induced short exact sequence

$$
0 \rightarrow R \stackrel{\alpha}{\rightarrow} M \rightarrow Q \rightarrow 0 .
$$

Let $\epsilon \in \operatorname{Ext}_{R}^{1}(Q, R)$ be the element corresponding to this short exact sequence via the Yoneda correspondence. Use $(-)^{\vee}$ to denote the operation $\operatorname{Hom}_{R}(-, R)$. If $P$. is a projective resolution of $Q$ consisting of finitely generated projective modules $P_{i}$, then we will say that $\epsilon$ is দ-phantom if a cocycle representing $\epsilon$ in $P_{1}^{\vee}$ is in $\operatorname{Im}\left(P_{0}^{\vee} \rightarrow P_{1}^{\vee}\right)_{P_{1}^{\vee}}^{\natural}$. We will call $\alpha$ a $\natural$-phantom extension of $R$ if $\epsilon$ is -phantom.

Discussion 2.3. Since the choice of projective resolution above is not canonical, we must demonstrate that whether $\epsilon \in \operatorname{Ext}_{R}^{1}(Q, R)$ is phantom or not is independent of the choice of $P_{\bullet}$. Let $Q$ • be another projective resolution of $Q$ consisting of finitely generated projective modules. Continue using $(-)^{\vee}$ to denote $\operatorname{Hom}_{R}(-, R)$.

Given $\epsilon$ in $\operatorname{Ext}_{R}^{1}(Q, R)$ representing the short exact sequence

$$
0 \rightarrow R \stackrel{\alpha}{\rightarrow} M \rightarrow Q \rightarrow 0,
$$

let $\phi \in P_{1}^{\vee}$ and $\phi^{\prime} \in Q_{1}^{\vee}$ be corresponding cocycles. Given $\phi \in \operatorname{Im}\left(P_{0}^{\vee} \rightarrow P_{1}^{\vee}\right)_{P_{1}^{\vee}}^{\natural}$, we will show that $\phi^{\prime} \in \operatorname{Im}\left(Q_{0}^{\vee} \rightarrow Q_{1}^{\vee}\right)_{Q_{1}^{\vee}}^{\natural}$ as well. 
We can lift the identity map $Q \stackrel{\text { id }}{\rightarrow} Q$ to a map of complexes as follows:

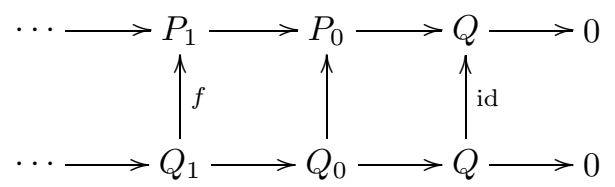

Applying $(-)^{\vee}$ to $(\#)$ yields the commutative diagram

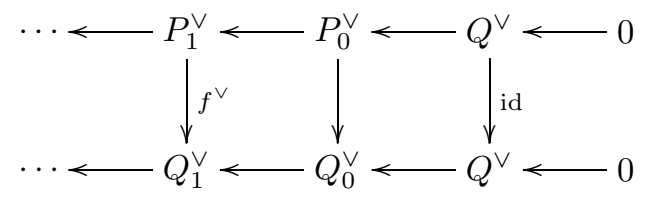

Via the Yoneda correspondence, $\phi \mapsto \phi^{\prime}$ under the map $f^{\vee}$. Since the element $\phi$ is in $\operatorname{Im}\left(P_{0}^{\vee} \rightarrow P_{1}^{\vee}\right)_{P_{1}^{\vee}}^{\natural}$, applying Axiom (4) to $f^{\vee}$ gives us

$$
\phi^{\prime}=f^{\vee}(\phi) \in \operatorname{Im}\left(P_{0}^{\vee} \rightarrow Q_{1}^{\vee}\right)_{Q_{1}^{\vee}}^{\natural}
$$

Since $\operatorname{Im}\left(P_{0}^{\vee} \rightarrow Q_{1}^{\vee}\right) \subseteq \operatorname{Im}\left(Q_{0}^{\vee} \rightarrow Q_{1}^{\vee}\right)$, Axiom (3) shows that $\phi^{\prime}$ is in $\operatorname{Im}\left(Q_{0}^{\vee} \rightarrow\right.$ $\left.Q_{1}^{\vee}\right)_{Q_{1}^{\vee}}^{\natural}$, as claimed.

Discussion 2.4. Now that we have a well-defined notion of $\downarrow$-phantom extensions, we demonstrate more explicitly what it means for a module to be t-phantom. Let $(R, \mathfrak{m})$ be a complete local domain with a closure operation satisfying the axioms. For a finitely generated $R$-module $M$ and an injection $R \stackrel{\alpha}{\rightarrow} M$, if we set $Q=$ $M / \alpha(R)$, we have the short exact sequence

$$
0 \rightarrow R \stackrel{\alpha}{\rightarrow} M \rightarrow Q \rightarrow 0
$$

Let $w_{1}=\alpha(1)$, and let $w_{2}, \ldots, w_{n}$ be elements of $M$ such that the images $\overline{w_{2}}, \ldots, \overline{w_{n}}$ in $Q$ form a minimal generating set for $Q$. Then $w_{1}, \ldots, w_{n}$ generate $M$. Let

$$
R^{m} \stackrel{\nu}{\rightarrow} R^{n-1} \stackrel{\mu}{\rightarrow} Q \rightarrow 0
$$

be a minimal free presentation of $Q$, where $R^{n-1}$ has basis $f_{2}, \ldots, f_{n}$ such that $\mu$ is given by $f_{i} \mapsto \overline{w_{i}}$. We can also choose a basis for $R^{m}$ such that $\nu$ is given by the $(n-1) \times m$ matrix

$$
\nu:=\left(\begin{array}{ccc}
b_{21} & \cdots & b_{2 m} \\
\vdots & \ddots & \vdots \\
b_{n 1} & \cdots & b_{n m}
\end{array}\right)
$$

where the entries $b_{i j}$ are in $\mathfrak{m}$. We can then construct the diagram

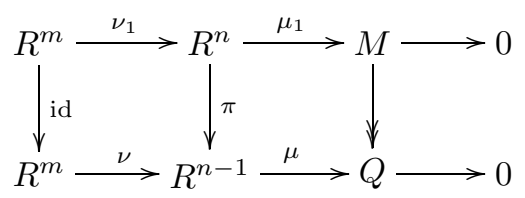


where $R^{n}$ has basis $\epsilon_{1}, \ldots, \epsilon_{n}, \pi\left(\epsilon_{i}\right)=f_{i}$ for $i>1$, and $\pi\left(\epsilon_{1}\right)=0$. The map $\mu_{1}$ is given by $\epsilon_{i} \mapsto w_{i}$, and $\nu_{1}$ is given by the $n \times m$ matrix

$$
\nu_{1}:=\left(\begin{array}{ccc}
b_{11} & \cdots & b_{1 m} \\
b_{21} & \cdots & b_{2 m} \\
\vdots & \ddots & \vdots \\
b_{n 1} & \cdots & b_{n m}
\end{array}\right)=\left(\begin{array}{ccc}
b_{11} & \cdots & b_{1 m} \\
& \nu &
\end{array}\right),
$$

where $b_{1 j} w_{1}+b_{2 j} w_{2}+\cdots+b_{n j} w_{n}=0$ in $M$, for $1 \leq j \leq m$. (Such $b_{1 j}$ exist because $b_{2 j} \overline{w_{2}}+\cdots+b_{n j} \overline{w_{n}}=0$ in $Q=M / R w_{1}$.)

From the construction, it is clear that (2.7) commutes and that $\mu_{1} \circ \nu_{1}$ is the zero map. The choice of the $w_{i}$ implies that (2.7) is exact at $M$. To see that $\operatorname{ker} \mu_{1} \subseteq \operatorname{Im} \nu_{1}$, suppose that $r_{1} w_{1}+\cdots r_{n} w_{n}=0$ in $M$. Then $r_{2} \overline{w_{2}}+\cdots+r_{n} \overline{w_{n}}=0$ in $Q$, and so there exist $s_{1}, \ldots, s_{m}$ in $R$ such that

$$
\nu\left[\left(s_{1}, \ldots, s_{m}\right)^{\operatorname{tr}}\right]=\left(r_{2}, \ldots, r_{n}\right)^{\operatorname{tr}},
$$

where $(-)^{\operatorname{tr}}$ denotes the transpose of a matrix. Then

$$
\nu_{1}\left[\left(s_{1}, \ldots, s_{m}\right)^{\mathrm{tr}}\right]=\left(r, r_{2}, \ldots, r_{n}\right)^{\mathrm{tr}} \in R^{n} .
$$

Since $\mu_{1} \circ \nu_{1}=0$, we see that $r w_{1}+r_{2} w_{2}+\cdots+r_{n} w_{n}=0$ in $M$. Therefore $r w_{1}=r_{1} w_{1}$, and so

$$
\alpha\left(r-r_{1}\right)=\left(r-r_{1}\right) \alpha(1)=\left(r-r_{1}\right) w_{1}=0 .
$$

Since $\alpha$ is injective, $r=r_{1}$ so that the vector $\left(r_{1}, \ldots, r_{n}\right)^{\operatorname{tr}}$ is in $\operatorname{Im} \nu_{1}$.

We can now conclude that the top row of (2.7) is a finite free presentation of $M$. (Since we do not know a priori whether $w_{1}, \ldots, w_{n}$ form a minimal basis for $M$, we cannot say whether our presentation of $M$ is minimal.)

We also obtain a commutative diagram with exact rows:

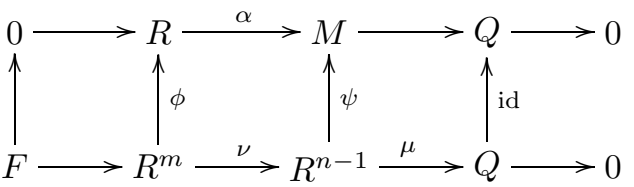

where $F$ is free, $\psi\left(f_{2}\right)=w_{2}, \ldots, \psi\left(f_{n}\right)=w_{n}$, and $\phi$ is given by the $1 \times m$ matrix $\left(-b_{11}, \ldots,-b_{1 m}\right)$. Because $\alpha$ is injective and $b_{1 j} w_{1}+\cdots+b_{n j} w_{n}=0$ in $M$ for $1 \leq j \leq m$, it is clear that (2.8) commutes as claimed. We can then take the dual into $R$ of (2.8):

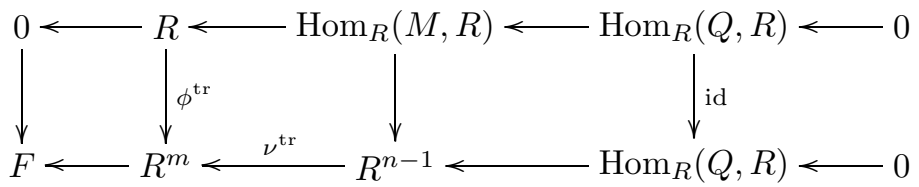

Let $Z$ be the kernel of $R^{m} \cong \operatorname{Hom}_{R}\left(R^{m}, R\right) \rightarrow \operatorname{Hom}_{R}(F, R) \cong F$, and let $B$ be the image of $\nu^{\mathrm{tr}}$. Then an element of $\operatorname{Ext}_{R}^{1}(Q, R)$ is an element of $Z / B$. As described in [HH3, Discussion 5.5], the element of $\operatorname{Ext}_{R}^{1}(Q, R)$ corresponding to the short exact sequence (2.5) is represented by the map $\phi: R^{m} \rightarrow R$ in (2.8). Equivalently, it is represented by the image of $\phi^{\text {tr }}$ in (2.9).

We can now state an equivalent condition for a finitely generated $R$-module $M$ to be $\bullet$-phantom. 
Lemma 2.10. Let $(R, \mathfrak{m})$ be a complete local domain possessing a closure operation satisfying Axioms (1)-(5). Let $M$ be a finitely generated module, and let $\alpha: R \rightarrow$ $M$ be an injective map. Using the notation of Discussion 2.4, $\alpha$ is a 4 -phantom extension of $R$ if and only if the vector $\left(b_{11}, \ldots, b_{1 m}\right)^{\text {tr }}$ is in $B_{R^{m}}^{\natural}$, where $B$ is the $R$-span in $R^{m}$ of the vectors $\left(b_{i 1}, \ldots, b_{i m}\right)^{\text {tr }}$ for $2 \leq i \leq n$, the $b_{i j}$ are in $\mathfrak{m}$ for $2 \leq i \leq n$ and all $j$, and $(-)^{\operatorname{tr}}$ denotes transpose.

Proof. By our definition and the constructions above, $\alpha$ is $\downarrow$-phantom if and only if the cocycle representing the corresponding element $\epsilon$ in $\operatorname{Ext}_{R}^{1}(Q, R)$ is in $\left(\operatorname{Im} \nu^{\operatorname{tr}}\right)_{R^{m}}^{\natural}$. Recall that $\epsilon$ is represented by the image of $\phi^{\text {tr }}$, which is $\left(-b_{11}, \ldots,-b_{1 m}\right)^{\text {tr }}$. Moreover, the image of $\nu^{\text {tr }}$ is the $R$-span of the row vectors of $\nu$, which is the $R$-span of $\left(b_{i 1}, \ldots, b_{i m}\right)^{\text {tr }}$ for $2 \leq i \leq n$. The claim concerning the $b_{i j}$ also follows from the preceding discussion.

We can now prove the following fact, an analogue of [HH3, Proposition 5.14], by including Axiom (6).

Lemma 2.11. Let $(R, \mathfrak{m})$ be a complete local domain possessing a closure operation satisfying Axioms (1)-(6). Let $M$ be a finitely generated $R$-module. If $\alpha: R \rightarrow M$ is a -phantom extension, then $\alpha(1) \notin \mathfrak{m} M$.

Proof. Suppose that $\alpha(1) \in \mathfrak{m} M$. Using the notation from Discussion 2.4, we have $\alpha(1)=w_{1}$. So, $\alpha(1) \in \mathfrak{m} M$ if and only if $w_{1}=r_{2} w_{2}+\cdots+r_{n} w_{n}$ such that the $r_{i}$ are in $\mathfrak{m}$. This occurs if and only if the vector $\left(1,-r_{2}, \ldots,-r_{n}\right)^{\operatorname{tr}}$ is in $\operatorname{Im} \nu_{1}$. In order for a vector with first component a unit to be in $\operatorname{Im} \nu_{1}$, the first row of $\nu_{1}$ must generate the unit ideal, i.e., $\left(b_{11}, \ldots, b_{1 m}\right) R=R$. Therefore, there exists $j_{0}$ such that $b_{1 j_{0}} \in R \backslash \mathfrak{m}$. By Lemma 2.10, since $\alpha$ is -phantom, the vector $\left(b_{11}, \ldots, b_{1 m}\right)^{\operatorname{tr}}$ is in $B_{R^{m}}^{\natural}$, where $B$ is the $R$-span in $R^{m}$ of the vectors $\left(b_{i 1}, \ldots, b_{i m}\right)^{\text {tr }}$ for $2 \leq i \leq n$. Since $b_{i j} \in \mathfrak{m}$ for $2 \leq i \leq n$ and $1 \leq j \leq m$, Axiom (3) implies that $\left(b_{11}, \ldots, b_{1 m}\right)^{\operatorname{tr}}$ is in $\left(\mathfrak{m} R^{m}\right)_{R^{m}}^{\natural}$. Using Axiom (4), for the projection $R^{m} \rightarrow R$ mapping onto the $j_{0}^{t h}$-coordinate, we see that $b_{1 j_{0}} \in(\mathfrak{m} R)_{R}^{\natural}$, but $(\mathfrak{m} R)_{R}^{\natural}=\mathfrak{m}$ by Axiom (6). This implies that $b_{1 j_{0}}$ cannot be a unit, and so $\alpha(1) \notin \mathfrak{m} M$.

\section{The axioms induce Balanced Big Cohen-Macaulay modules}

In order to use -phantom extensions and our axioms to produce balanced big Cohen-Macaulay modules, we will use module modifications as found in [Ho1] and HH3. We will leave it to the reader to review the details of the construction in

[HH3, Discussion 5.15], but we will outline the idea and its connection to $\downarrow$-phantom extensions.

Discussion 3.1. We can produce a balanced big Cohen-Macaulay module by starting with $R$ and successively modifying it to trivialize any relations on systems of parameters. Starting with $M_{0}=R$ and $w_{0}=1$, construct a sequence of modules $M_{t}$ containing elements $w_{t}$ and homomorphisms $M_{t} \rightarrow M_{t+1}$ such that $w_{t} \mapsto w_{t+1}$. Given a module $M_{t}$, we construct $M_{t+1}$ by selecting a relation $x_{k+1} u=x_{1} u_{1}+\cdots+x_{k} u_{k}$ in $M_{t}$, where $x_{1}, \ldots, x_{k+1}$ forms a partial system of parameters in $R$. Then define

$$
M_{t+1}:=\frac{M_{t} \oplus R f_{1} \oplus \cdots \oplus R f_{k}}{R\left(-u \oplus x_{1} f_{1} \oplus \cdots \oplus x_{k} f_{k}\right)}
$$


in order to trivialize the relation in $M_{t+1}$. The construction yields a natural map $M_{t} \rightarrow M_{t+1}$, where we define $w_{t+1}$ to be the image of $w_{t}$. Since we started the chain with $R$ and 1 , we also have maps $R \rightarrow M_{t}$ with $1 \mapsto w_{t}$ for all $t$.

Continuing in this manner, we will define the module $B$ to be the direct limit of all such $M_{t}$. The module $B$ has the property that all relations on parameters in $B$ are trivial, but it is a priori unclear whether or not we have trivialized too much and caused $\mathfrak{m} B=B$. To avoid this possibility, we will show that the image of 1 from $R$ in $B$ is not in $\mathfrak{m} B$ by showing that $w_{t} \notin \mathfrak{m} M_{t}$ for all $t$.

In order to accomplish this goal, we will demonstrate that each module modification $R \rightarrow M_{t}$ is a -phantom extension. By Lemma 2.11, we then see that $w_{t} \notin \mathfrak{m} M_{t}$ for all $t$, proving that $B$ is a balanced big Cohen-Macaulay module.

Discussion 3.2. Let $R$ be a complete local domain. Let $\alpha: R \rightarrow M$ be an injective map to a finitely generated $R$-module $M$ with a relation

$$
x_{1} u_{1}+\cdots+x_{k+1} u_{k+1},
$$

where $x_{1}, \ldots, x_{k+1}$ is part of a system of parameters for $R$. (We will show in Lemma 3.8 that $R$ injects into all module modifications and use the identity map on $R$ as a base case.)

Without loss of generality, denote the (not necessarily minimal) generators of $M$ by

$$
m_{1}=: \alpha(1), m_{2}, \ldots, m_{n-k-1}, m_{n-k}:=u_{1}, \ldots, m_{n}:=u_{k+1} .
$$

We then have a short exact sequence as in (2.5):

$$
0 \rightarrow R \stackrel{\alpha}{\rightarrow} M \rightarrow Q \rightarrow 0,
$$

where $Q:=M / \alpha(R)=M / R m_{1}$. Using the matrices worked out for the diagram (2.7), we obtain

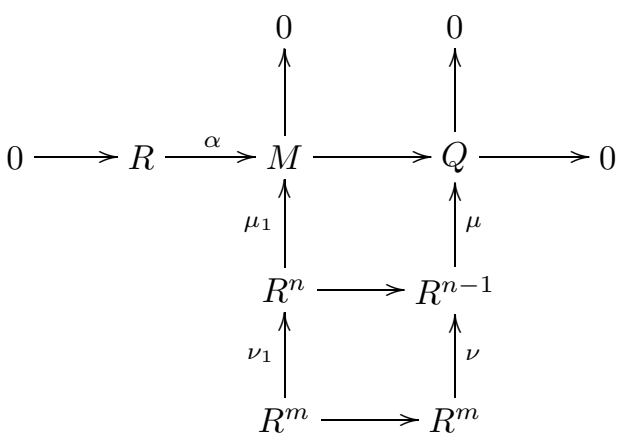

where $\mu$ and $\mu_{1}$ are as given in Discussion 2.4.

$$
\nu:=\left(\begin{array}{cccc}
b_{21} & \cdots & b_{2, m-1} & 0 \\
\vdots & \ddots & \vdots & \vdots \\
b_{n-k-1,1} & \cdots & b_{n-k-1, m-1} & 0 \\
b_{n-k, 1} & \cdots & b_{n-k, m-1} & x_{1} \\
\vdots & \ddots & \vdots & \vdots \\
b_{n 1} & \cdots & b_{n, m-1} & x_{k+1}
\end{array}\right)
$$


(as we can include the relation (3.3) without loss of generality), and

$$
\nu_{1}:=\left(\begin{array}{cccc}
b_{11} & \cdots & b_{1, m-1} & 0 \\
& & \nu &
\end{array}\right) .
$$

Then (3.4) gives a commutative diagram with exact rows and columns and free presentations of $M$ and $Q$.

Notation 3.5. Using the construction in Discussion 3.2 , we define the following:

$\mathbf{x}:=\left(b_{11}, \ldots, b_{1, m-1}, 0\right)^{\mathrm{tr}}$, the transpose of the first row of $\nu_{1}$.

$\mathbf{y}:=\left(b_{n 1}, \ldots, b_{n, m-1}, x_{k+1}\right)^{\mathrm{tr}}$, the transpose of the last row of $\nu$.

$H:=R$-module generated by all the rows of $\nu$ except the last row.

$I:=\left(x_{1}, \ldots, x_{k}\right)$.

Adapting the characterization of $\downarrow$-phantom extensions using free presentations from Lemma 2.10, we have

Lemma 3.6. Let $R$ be a complete local domain possessing a closure operation satisfying Axioms (1)-(5). Use the notation from Discussion 3.2 and Notation 3.5 . The map $\alpha$ is 4 -phantom if and only if

$$
\mathbf{x} \in(R \mathbf{y}+H)_{R^{m}}^{\natural} .
$$

Discussion 3.7. We now investigate module modifications. Using the notation developed in Discussion 3.2, use free generators $f_{1}, \ldots f_{k}$ to define the modification

$$
M^{\prime}:=\frac{M \oplus R f_{1} \oplus \cdots \oplus R f_{k}}{R\left(m_{n} \oplus x_{1} f_{1} \oplus \cdots \oplus x_{k} f_{k}\right)}
$$

of $M$ with respect to the relation $(3.3)$ on the parameters $x_{1}, \ldots, x_{k+1}$. Define

$$
\alpha^{\prime}: R \rightarrow M^{\prime}
$$

as the composition $\alpha^{\prime}=\beta \circ \alpha$, where $\beta(u)$ is the image of $u \oplus 0 \cdots \oplus 0$ in $M^{\prime}$ for all $u \in M$.

As stated in Discussion 3.1 in order to show that our sequence of modifications leads to a balanced big Cohen-Macaulay module $B$, it suffices to show that $\alpha^{\prime}$ is ฤ-phantom when $\alpha$ is $\natural$-phantom.

Lemma 3.8. Using the constructions given in Discussions 3.2 and [3.7, the map $\alpha^{\prime}$ is injective.

Proof. Since $\alpha^{\prime}=\beta \circ \alpha$ and $\alpha$ is injective, it suffices to show that $\beta$ is also injective. If $\beta(u)=0$ in $M^{\prime}$, then

$$
u \oplus 0 \oplus \cdots \oplus 0=r\left(m_{n} \oplus x_{1} f_{1} \oplus \cdots \oplus x_{k} f_{k}\right)
$$

so that $r x_{1}=0$. As $R$ is a domain, $r=0$ and thus $u=r m_{n}=0$.

One can construct free presentations for $M^{\prime}$ and $Q^{\prime}:=M^{\prime} / \alpha^{\prime}(1)$ similarly to how we constructed free presentations for $M$ and $Q$ in (3.4) because we added $k$ new generators and one new relation. 
Lemma 3.9. The following diagram is commutative and gives free presentations for $M^{\prime}$ and $Q^{\prime}$ :

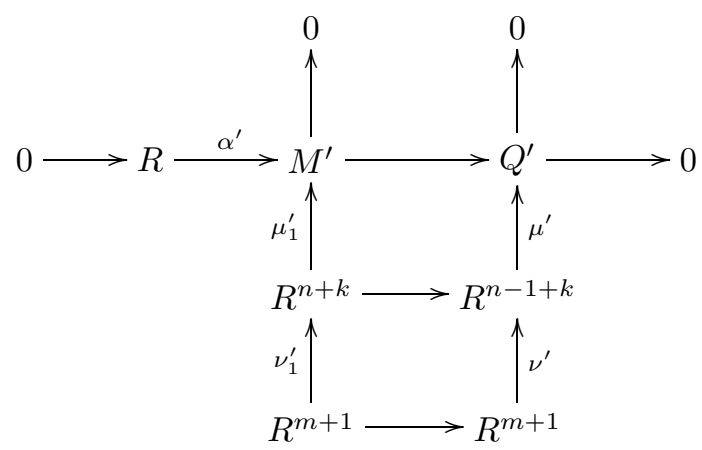

where the matrices $\mu^{\prime}$ and $\mu_{1}^{\prime}$ are extensions of $\mu$ and $\mu_{1}$ that account for the $k$ new generators $w_{1}, \ldots, w_{k}, \nu^{\prime}$ is the $(n-1+k) \times(m+1)$ matrix

$$
\nu^{\prime}:=\left(\begin{array}{c|c} 
& 0 \\
\nu & \vdots \\
& 0 \\
\hline & x_{1} \\
\mathbf{0} & \vdots \\
& x_{k}
\end{array}\right),
$$

and $\nu_{1}^{\prime}$ is the $(n+k) \times(m+1)$ matrix

$$
\nu_{1}^{\prime}:=\left(\begin{array}{ccccc}
b_{11} & \cdots & b_{1, m-1} & 0 & 0 \\
& & \nu^{\prime} & &
\end{array}\right) .
$$

Proof. All of the claims are straightforward to verify. We will describe the exactness at $R^{n+k}$ though. Indeed, $\mu_{1}^{\prime} \circ \nu_{1}^{\prime}=0$ as $\mu_{1} \circ \nu_{1}=0$ and $m_{n}+x_{1} w_{1}+\cdots+x_{k} w_{k}=0$ in $M^{\prime}$. To see why the kernel of $\mu_{1}^{\prime}$ is contained in the image of $\nu_{1}^{\prime}$, suppose that $\mu_{1}^{\prime}\left(r_{1}, \ldots, r_{n+k}\right)^{\mathrm{tr}}=0$. Then

$$
r_{1} m_{1}+\cdots r_{n} m_{n}+r_{n+1} w_{1}+\cdots+r_{n+k} w_{k}=r\left(m_{n}+x_{1} w_{1}+\cdots+x_{k} w_{k}\right)
$$

in $M \oplus R^{k}$. Thus,

$$
r_{1} m_{1}+\cdots+r_{n-1} m_{n-1}+\left(r_{n}-r\right) m_{n}=0
$$

in $M$ and $r_{n+i}=r x_{i}$ for all $1 \leq i \leq k$. Thus, $\left(r_{1}, \ldots, r_{n+k}\right)^{\text {tr }}$ is an $R$-linear combination of the columns of $\nu_{1}^{\prime}$.

We now characterize when $\alpha^{\prime}$ is $\sharp$-phantom. The proof follows directly from Lemma 2.10 and our computations of $\nu_{1}^{\prime}$ and $\nu^{\prime}$.

Lemma 3.11. Let $R$ be a complete local domain possessing a closure operation satisfying Axioms (1)-(5). Use Notation 3.5. The map $\alpha^{\prime}$ is 4 -phantom if and only if

$$
\mathbf{x} \oplus 0 \in(R(\mathbf{y} \oplus 1)+(H \oplus 0)+I(\mathbf{0} \oplus 1))_{R^{m+1}}^{\natural} .
$$

We now apply Axiom (6) to connect the conditions characterizing when $\alpha$ and $\alpha^{\prime}$ are t-phantom. 
Lemma 3.12. Let $R$ be a complete local domain possessing a closure operation satisfying Axioms (1)-(6). Use Notation 3.5. Let $\mathbf{v}$ be in $R^{m}$. If $\mathbf{v} \in(I \mathbf{y}+H)_{R^{m}}^{\natural}$, then $\mathbf{v} \oplus 0 \in(R(\mathbf{y} \oplus 1)+(H \oplus 0)+I(\mathbf{0} \oplus 1))_{R^{m+1}}^{\natural}$.

Proof. By Axiom (6), $0_{R}^{\natural}=0$. By Lemma 1.2(b),

$$
(I(\mathbf{y} \oplus 0)+(H \oplus 0))_{R^{m+1}}^{\natural}=(I \mathbf{y}+H)_{R^{m}}^{\natural} \oplus 0_{R}^{\natural}=(I \mathbf{y}+H)_{R^{m}}^{\natural} \oplus 0 .
$$

Since $I(\mathbf{y} \oplus 0) \subseteq R(\mathbf{y} \oplus 1)+I(\mathbf{0} \oplus 1)$, Axiom (3) finishes the proof.

Proposition 3.13. Let $R$ be as in the previous lemma. Use Notation 3.5. If $\mathbf{x} \in(I \mathbf{y}+H)_{R^{m}}^{\natural}$, then $\alpha^{\prime}$ is $\natural$-phantom.

Before we apply Axiom (7) (the generalized colon-capturing property), we look again at the closure conditions found in Lemma 3.6 and Proposition 3.13 .

Lemma 3.14. Let $R$ be a complete local domain possessing a closure operation satisfying Axioms (1)-(5). Use Notation 3.5, let $Q:=R^{m} / H$, let $v:=\mathbf{y}+H$, and let $\mathbf{a} \in R^{m}$.

(a) $\mathbf{a} \in(R \mathbf{y}+H)_{R^{m}}^{\natural}$ if and only if $\mathbf{a}+H \in(R v)_{Q}^{\natural}$.

(b) $\mathbf{a} \in(I \mathbf{y}+H)_{R^{m}}^{\natural}$ if and only if $\mathbf{a}+H \in(I v)_{Q}^{\natural}$.

We can now achieve the goal we set at the end of Discussion 3.7

Proposition 3.15. Let $R$ be a complete local domain possessing a closure operation satisfying Axioms (1)-(7). Use Notation 3.5. Let $Q:=R^{m} / H$, and let $v:=\mathbf{y}+H$. If $\alpha$ is $\natural$-phantom, then $\alpha^{\prime}$ is $\natural$-phantom.

Proof. By Lemma 3.6 and the last lemma, $\alpha$ is 4 -phantom if and only if $\mathbf{x}+H \in$ $(R v)_{Q}^{\natural}$. Let $\pi: R^{m} \rightarrow R / I$ be the surjection that projects an element of $R^{m}$ onto its last coordinate modulo $I$. Since the last entries of the generators of $H$ exactly generate $I=\left(x_{1}, \ldots, x_{k}\right)$, we see that $\pi(H)=I$, and so $\pi$ factors through $Q$. Let $f: Q \rightarrow R / I$ be the resulting surjection. As the last entry of $\mathbf{x}$ is 0 , we have that $\mathbf{x}+H$ is also in the kernel of $f$. By Axiom (7), $\mathbf{x}+H \in(I v)_{Q}^{\natural}$ and so $\mathbf{x} \in(I \mathbf{y}+H)_{R^{m}}^{\natural}$, which implies that $\alpha^{\prime}$ is -phantom by Proposition 3.13 ,

Since $\alpha: R \rightarrow M$ being Ł-phantom implies that the map $\alpha^{\prime}: R \rightarrow M^{\prime}$ is phantom, where $M^{\prime}$ is a module modification of $M$, we can conclude the following theorem by using Lemma 2.11 and Discussions 3.1 and 3.7.

Theorem 3.16. Let $R$ be a complete local domain possessing a closure operation that satisfies Axioms (1)-(7). Then there exists a balanced big Cohen-Macaulay $R$-module $B$.

\section{Big Cohen-Macaulay modules imply the aXioms}

In this section, assume that $(R, \mathfrak{m})$ is a complete local domain and that $B$ is a balanced big Cohen-Macaulay $R$-module. We will define a closure operation for $R$-modules, based on $B$, that will satisfy Axioms 1.1.

Definition 4.1. Given $R$ and $B$ as above and finitely generated $R$-modules $N \subseteq M$, define $N_{M}^{\natural}$ to be the set of all $u \in M$ such that for all $b \in B$ we have $b \otimes u \in$ $\operatorname{Im}(B \otimes N \rightarrow B \otimes M)$. 
Theorem 4.2. The closure operation $N_{M}^{\natural}$ defined above in terms of the balanced big Cohen-Macaulay R-module B satisfies Axioms 1.1.

Proof.

(1) The claim that $N \subseteq N_{M}^{\natural}$ is clear and basic tensor product properties imply that $N_{M}^{\natural}$ is a submodule of $M$.

(2) By Axiom (1), $N_{M}^{\natural} \subseteq\left(N_{M}^{\natural}\right)_{M}^{\natural}$. Suppose that $u \in\left(N_{M}^{\natural}\right)_{M}^{\natural}$, and let $b \in B$. Then $b \otimes u \in \operatorname{Im}\left(B \otimes N_{M}^{\natural} \rightarrow B \otimes M\right)$, which implies that $b \otimes u=\sum_{j} b_{j} \otimes v_{j}$ in $B \otimes M$, where $b_{j} \in B$ and $v_{j} \in N_{M}^{\natural}$. Since each $b_{j} \otimes v_{j} \in \operatorname{Im}(B \otimes N \rightarrow B \otimes M)$, we have $b \otimes u \in \operatorname{Im}(B \otimes N \rightarrow B \otimes M)$. Thus, $u \in N_{M}^{\natural}$.

(3) Given $N \subseteq M \subseteq W$, the inclusion $N_{W}^{\natural} \subseteq M_{W}^{\natural}$ follows from the fact that $\operatorname{Im}(B \otimes N \rightarrow B \otimes W) \subseteq \operatorname{Im}(B \otimes M \rightarrow B \otimes W)$.

(4) Given $N \subseteq M$ and $f: M \rightarrow W$, the inclusion $f\left(N_{M}^{\natural}\right) \subseteq f(N)_{W}^{\natural}$ follows from the fact that the map $B \otimes f$ maps $\operatorname{Im}(B \otimes N \rightarrow B \otimes M)$ to $\operatorname{Im}(B \otimes f(N) \rightarrow$ $B \otimes W)$.

(5) Suppose that $N_{M}^{\natural}=N$ and that $\bar{u} \in 0_{M / N}^{\natural}$. Then for all $b \in B$, we have $b \otimes \bar{u}=0$ in $B \otimes M / N \cong(B \otimes M) / \operatorname{Im}(B \otimes N \rightarrow B \otimes M)$. Thus, $b \otimes u \in$ $\operatorname{Im}(B \otimes N \rightarrow B \otimes M)$ so that $u \in N_{M}^{\natural}=N$. Therefore, $\bar{u}=0$ in $M / N$.

(6) Suppose that $x \in \mathfrak{m}_{R}^{\natural} \backslash \mathfrak{m}$. Then $x$ is a unit in $R$, and for all $b \in B$ we have $x b \in \mathfrak{m} B$. Therefore, $x$ annihilates $B / \mathfrak{m} B$, but since $x$ is a unit, $B=\mathfrak{m} B$, contradicting the fact that $B$ is a balanced big Cohen-Macaulay $R$-module.

Now, suppose that $x \in 0_{R}^{\natural}$. Then $x b=0$ in $B$ for all $b \in B$. Since $R$ is a domain and $B$ is a balanced big Cohen-Macaulay $R$-module, $x$ cannot annihilate nonzero elements of $B$ unless $x=0$.

(7) Suppose $f: M \rightarrow R / I$ (we do not need to assume it is surjective), where $x_{1}, \ldots, x_{k+1}$ is a partial system of parameters for $R, I=\left(x_{1}, \ldots, x_{k}\right)$, and $f(v)=x_{k+1}+I$. Let $u \in(R v)_{M}^{\natural} \cap \operatorname{ker} f$, and let $b \in B$. Then $f(u)=0$, and $b \otimes u=c_{b} \otimes v$ in $B \otimes M$. Therefore, $c_{b} \otimes f(v)=0$ in $B \otimes R / I$, which implies that $c_{b} x_{k+1}+I B=I B$ in $B / I B$. Hence, $c_{b} x_{k+1} \in I B$. Since $B$ is balanced big Cohen-Macaulay, $c_{b} \in I B$. Thus,

$$
b \otimes u \in \operatorname{Im}(I B \otimes R v \rightarrow B \otimes M)=\operatorname{Im}(B \otimes I v \rightarrow B \otimes M),
$$

and so $u \in(I v)_{M}^{\natural}$.

\section{Common Closure operations And the aXioms}

In the final section we compare tight closure, plus closure, Frobenius closure, and solid closure with the axioms. We refer the reader to [HH1, especially Section 8, for the definitions and notation involved in tight closure of modules. See $\mathrm{HH} 2$ and $[\mathrm{Sm}$ for the details on plus closure and its properties. See [HH3, Section 7] for information on Frobenius closure, and see [Ho2] for details on solid closure.

It is easy to see that any closure operation that can be defined in terms of tensor products with $R$-modules or $R$-algebras, e.g., plus closure, Frobenius closure, or solid closure, will satisfy Axioms (1)-(5). These three closure operations also satisfy Axiom (6) as each is contained in the integral closure. The crucial axiom to verify is thus Axiom (7). 
Example 5.1. Plus closure in prime characteristic $p$ satisfies Axiom (7) as it is defined in terms of the balanced big Cohen-Macaulay $R$-algebra $R^{+}$, the absolute integral closure of $R$.

Example 5.2. Frobenius closure does not satisfy Axiom (7) in general as it does not even satisfy colon-capturing. For example, let $K$ be a field of prime characteristic $p \equiv 1(\bmod 3)$. Set $R=K[X, Y, Z] /\left(X^{3}+Y^{3}+Z^{3}\right)=K[x, y, z]$, the cubical cone, and set $S=K[s, t]$. Then let $T=K[x s, y s, z s, x t, y t, z t]$ be the Segre product of $R$ and $S$. After localization at the maximal ideal and completion, $\widehat{T}$ is a complete local domain that is not Cohen-Macaulay. It can be checked that $y s, x t$, and $x s-y t$ form a system of parameters, but the relation $(z s)(z t)(x s-y t)=(z s)^{2}(x t)-(z t)^{2}(y s)$ shows that we do not have a regular sequence. (See [Ho3 pp. 15-16] for the details.) On the other hand, $R$ is $F$-pure (see [Ho3, pp. 162, 269]) and so is $R[s, t]$. Since $T$ is a direct summand of $R[s, t]$ as a $T$-module, $T$ and $\widehat{T}$ are also $F$-pure. Therefore, $I^{F}=I$ for all ideals in $\widehat{T}$ and so colon-capturing cannot hold in $\widehat{T}$ since it is not Cohen-Macaulay.

Example 5.3. In positive characteristic, solid closure and tight closure coincide for complete local domains [Ho2, Theorem 8.6]. Proposition 5.6 below will then show that solid closure satisfies Axiom (7) in this case. In equal characteristic 0, solid closure contains the various notions of tight closure in equal characteristic 0 [ $\mathrm{Ho} 2$, Theorem 11.4]. Since those tight closure operations capture colons, solid closure does as well. Whether or not solid closure obeys Axiom (7) in characteristic 0 is unknown. In mixed characteristic, the situation is more mysterious. It is not even known whether a complete local domain having all parameter ideals solidly closed must be Cohen-Macaulay for dimension greater than two, and so colon-capturing may not hold.

Example 5.4. In positive characteristic, tight closure satisfies all of Axioms 1.1 for a complete local domain $R$. Axioms (1), (2), and (3) are given in HH1, Proposition 8.5(a), (e), (b)]. Axiom (5) is found in [HH1, Remark 8.4]. Axiom (6) is a consequence of [HH1, Theorem 5.2] since 0 and $\mathfrak{m}$ are integrally closed in $R$.

For lack of a convenient reference, we will outline the proof for Axiom (4) before proving Axiom (7), the generalized colon-capturing property.

Lemma 5.5. Let $R$ be any ring of prime characteristic $p>0$. Then Axiom (4) holds, i.e., given $f: M \rightarrow W$ with $N \subseteq M$, we have $f\left(N_{M}^{*}\right) \subseteq f(N)_{W}^{*}$.

Proof. If $u \in N_{M}^{*}$, then there exists a $c \in R$ not in any minimal prime such that $c \otimes u \in \operatorname{Im}\left(\mathbf{F}^{e}(N) \rightarrow \mathbf{F}^{e}(M)\right)$. Thus, $c \otimes f(u) \in \operatorname{Im}\left(\mathbf{F}^{e}(f(N)) \rightarrow \mathbf{F}^{e}(W)\right)$, so $f(u) \in f(N)_{W}^{*}$.

Proposition 5.6. Let $R$ be a complete local domain of prime characteristic $p>0$. Axiom (7), the generalized colon-capturing property, holds for tight closure.

Proof. Let $f: M \rightarrow R / I$ be a homomorphism, where $x_{1}, \ldots, x_{k+1}$ is a partial system of parameters for $R, I=\left(x_{1}, \ldots, x_{k}\right)$, and $f(v)=x_{k+1}$. Suppose that $u \in(R v)_{M}^{*} \cap \operatorname{ker} f$. We need to show that $u \in(I v)_{M}^{*}$. Let $c$ be a test element for $R$, which exists by [HH1, Corollary 6.24]. Then for all $q=p^{e}$, we have $c u^{q} \in(R v)_{M}^{[q]}$. Applying $f$, we have $c f(u)^{q}=r_{q} x_{k+1}^{q}$ in $R / I^{[q]}$. Since $f(u)=0, r_{q} x_{k+1}^{q} \in I^{[q]}$ so 
that $r_{q} \in I^{[q]}: x_{k+1}^{q}$. By regular colon-capturing for tight closure [HH1, Theorem 4.7], $r_{q} \in\left(I^{[q]}\right)^{*}$ and so $c r_{q} \in I^{[q]}$ since $c$ is a test element. Therefore, $c^{2} u^{q}=c r_{q} x_{k+1}^{q} \in(I v)_{M}^{[q]}$ and so $u \in(I v)_{M}^{*}$.

Example 5.7. Using the usual reduction to characteristic $p$ arguments (see [HH4, specifically Theorem 4.1.7 on colon-capturing), one can deduce that equal characteristic 0 tight closure notions also obey Axioms (1)-(7).

\section{ACKNOWLEDGMENTS}

My thanks to Mel Hochster for several helpful conversations during the preparation of this manuscript and for pointing out the example for an $F$-pure but non-Cohen-Macaulay complete local domain. I also thank the anonymous referee for many helpful suggestions that improved the exposition of the paper.

\section{REFERENCES}

[D] G. Dietz, Big Cohen-Macaulay algebras and seeds, Trans. Amer. Math. Soc. 359 (2007), no. 12, 5959-5989. MR2336312 (2008h:13021)

[Ho1] M. HochSTER, Topics in the homological theory of modules over commutative rings, CBMS Regional Conf. Ser. in Math. 24, Amer. Math. Soc., Providence, RI, 1975. MR0371879 $(51: 8096)$

[Ho2] M. Hochster, Solid closure, in: Commutative Algebra: Syzygies, Multiplicities and Birational Algebra, Contemp. Math. 159, Amer. Math. Soc., Providence, RI, 1994, 103-172. MR.1266182 (95a:13011)

[Ho3] M. HochsTer, Foundations of tight closure theory, lecture notes available at http://www.math.lsa.umich.edu/ hochster/mse.html.

[HH1] M. Hochster and C. Huneke, Tight closure, invariant theory, and the Briançon-Skoda theorem, J. Amer. Math. Soc. 3 (1990), 31-116. MR1017784 (91g:13010)

[HH2] M. Hochster and C. Huneke, Infinite integral extensions and big Cohen-Macaulay algebras, Annals of Math. 135 (1992), 53-89. MR1147957 (92m:13023)

[HH3] M. Hochster and C. Huneke, Tight closure of parameter ideals and splitting in modulefinite extensions, J. Algebraic Geom. 3 (1994), no. 4, 599-670. MR1297848 (95k:13002)

[HH4] M. Hochster and C. Huneke, Tight Closure in Equal Characteristic Zero, preprint available at http://www.math.lsa.umich.edu/ hochster/msr.html.

[Sm] K.E. SMith, Tight closure of parameter ideals, Invent. Math. 115 (1994), 41-60. MR.1248078(94k:13006)

Department of Mathematics, Gannon University, Erie, Pennsylvania 16541

E-mail address: gdietz@member.ams.org 